\title{
Bounded acceleration capacity drop in a Lagrangian formulation of the kinematic wave model with vehicle characteristics and unconstrained overtaking
}

\author{
S.C. Calvert, M. Snelder, H. Taale, F.L.M. van Wageningen-Kessels and S.P. Hoogendoorn
}

\begin{abstract}
In this contribution a model-based analysis of the application of bounded acceleration in traffic flow is considered as a cause for the capacity drop. This is performed in a Lagrangian formulation of the kinematic wave model with general vehicle specific characteristics. Unconstrained overtaking is presumed, which allows a demonstration to be given of the influence that constraints in traffic flow may have on the capacity drop. An experimental case demonstrates that bounded acceleration in traffic flow with unconstrained overtaking has very limited effect on the capacity drop. This implies that the capacity drop when incurred through bounded acceleration must make use of (semi-)constrained traffic flow, in which variety in vehicle acceleration ability may also be required to increase inhomogeneity. This is an important conclusion as it further defines the conditions required for capacity drop. The application of a Lagrangian formulation with advection invariant combined with bounded acceleration is also novel. The contribution further shows that the application of bounded acceleration in the presented model is feasible, although adjustments are required to capture the capacity drop through bounded acceleration.
\end{abstract}

Keywords: traffic modelling; capacity drop; bounded acceleration; kinematic wave model; Lagrangian coordinates; invariant advection

\section{INTRODUCTION}

In this contribution a bounded acceleration approach is described in a Lagrangian formulation of the first order kinematic wave model with vehicle specific characteristics. The effect of the application of bounded acceleration with unconstrained overtaking on the capacity drop is also shown and discussed. Here unconstrained indicates the ability of vehicles to overtake and therefore not be constrained by slower predecessors.

Traditionally first order macroscopic models consider traffic flow based on principles laid in the LWR model [1,2] and allow general traffic flow features to be described. However basic first order models, also known as kinematic wave models (KWM), do not capture many of the detailed dynamics of traffic flow, such as the interaction between vehicles in congestion. This has previously been described in detail and gave birth to second order models [3-7]. However the simplicity of first order models is a major advantage over second order models and therefore extensions have been proposed to help capture more traffic dynamics while retaining much of the simplicity [8]. One such advancement is the introduction of bounded acceleration. Bounded

Manuscript received April 15th, 2015.

All authors are with the Delft University of Technology, department of Transport \& Planning, PO Box 5048, 2600GA, Delft, The Netherlands. S. C. Calvert, and M. Snelder are also with TNO, the Netherlands Organisation for Applied Scientific Research, The Netherlands. Email: s.c.calvert@tudelft.nl, maaike.snelder@tno.nl, h.taale@tudelft.nl, F.L.M.vanWageningen-Kessels-1@ tudelft.nl, s.p.hoogendoorn@tudelft.nl acceleration was previously introduced by Lebacque [9] and has been applied and further developed by various researchers [10-13]. The approach is relatively simple, but effective, and involves bounding the accelerative ability of vehicles by preventing speeds that exceed a pre-set acceleration value as vehicles propagate. In the basic kinematic wave models, vehicles may accelerate at an unrealistic speed. By bounding this acceleration, a more realistic description of real traffic flow is given adhering to the physical capabilities of vehicles. The effect is especially visible for acceleration of vehicles from low speeds such as out of congestion. In this contribution a Lagrangian formulation of the KWM is applied. Traditionally Eulerian coordinates are applied which state that for a specific time and location a flow, such as traffic, will pass with certain characteristics $[14,15]$. Lagrangian coordinates in contrast are not fixed in space, but are given the freedom to transform with the speed of the resulting flow. This can be described as particles in a flow whose position are explicitly denoted by the speed of vehicles in individual consecutive temporal cells. Therefore the coordinates follow the flow rather than the flow following the coordinates, which is especially advantageous for use with bounded acceleration as the speed of traffic is the resultant from the fundamental equation used in the Lagrangian system, rather than traffic flow. The use of Lagrangian coordinates also allows a vehicle specific invariant term to propagate along with the vehicles for which it is valid and thus avoids numerical diffusion of driver behaviour variables $[15,16]$. This vehicle specific invariant term is introduced in parallel research to this contribution, but is concisely described in the following section. It basically describes the differences in driver characteristics between vehicles.

It has been claimed that application of bounded acceleration can make it possible to model the capacity drop in first order models [17, 18]. The capacity drop is defined as the difference between the breakdown capacity and the discharge capacity on a section of road and can frequently be observed after traffic breakdown between observations in a critical undersaturated traffic state and an oversaturated traffic state. The occurrence of the capacity drop is generally attributed to the so called hysteresis effect [19-21]. The hysteresis effect occurs in part due to differing driving behaviour as vehicles enter and exit congested traffic states [22] and is most commonly captured in macroscopic models in second order formulations. In these models an additional equation is given that describes the dynamics of vehicle flow. There have also been attempts to include the capacity drop in first order models [17].

Commonly the capacity drop is included in first order models through an explicit reduction of the constrained flow around a bottleneck location. This however focusses on the effect rather than the cause. This furthermore leads to discrepancies 
in modelling boundaries due to assumptions that are made to allow flows to be constrained. In this contribution we further analyse the application of the capacity drop through a bounded acceleration of vehicles rather than a bounded flow. This is tested for traffic flow, in which vehicle groups always have the possibility to overtake without conceding a penalty. This is an important addition as it can show that lateral constraints are not required or that there is a necessity for a certain containment of vehicles to allow the capacity drop to occur.

To summarize, the main contributions are the demonstration of bounded acceleration in a Lagrangian formulation of the KWM with vehicle characteristics through an advection invariant and an exploration of bounded acceleration capacity drop in traffic with unconstrained overtaking. The rest of the contribution includes a description of the models for which the bounded acceleration is applied. This is performed in section 2. Section 3 gives a demonstration of the effectiveness and relevance of the bounded acceleration in the model, with the papers conclusions given in section 4 .

\section{MODEL DESCRIPTION}

\section{A. Kinematic wave model}

The applied model in this contribution is a first order kinematic wave model (KWM). The KWM captures aggregated traffic flows described as the propagation of traffic waves and the adhering traffic characteristics. The concept of modelling kinematic waves of traffic was first introduced by Lighthill, Whitham and Richards [1,2] and is therefore often referred to as the LWR model. Construction of the kinematic waves is achieved through use of the fundamental relationship of traffic flow which is generally described by the relationship between the density $\rho$ and the flow $q$ of traffic. The model further relies on the conservation equation and initial boundary conditions. The conservation equation and the fundamental relation are denoted by:

$$
\begin{aligned}
& \partial_{t} \rho+\partial_{x} q=0 \\
& q=Q(\rho)
\end{aligned}
$$

Where $\rho$ is the traffic density in time $t$

$q$ is the flow in space $x$

$Q(\rho)$ is the shape of the fundamental relation

\section{B. Lagrangian formulation}

The KWM is formulated in Lagrangian coordinates in this contribution, which has a number of advantages. It has been shown that this leads to a higher accuracy, as the error due to numerical diffusion is much less than in the traditionally applied Eulerian coordinates [15, 16, 23]. The Lagrangian system describes the coordinates of particles in a flow explicitly as a function of their speed. Therefore the coordinates follow the flow rather than the flow following the coordinates. When describing the KWM in Lagrangian coordinates the same equations are formulated in a slightly differently way. The conservation equation and the fundamental relation are now given as:

$$
\begin{aligned}
& \partial_{t} s+\partial_{n} v=0 \\
& v=V(\rho(s))
\end{aligned}
$$

Where $s$ is the mean space headway of vehicles in a single cell

$v$ is the mean speed of vehicles,

$n$ is the vehicles number, which decreases in the driving direction

$V(\rho(s))$ is the shape of the fundamental relation

The fundamental relation in Lagrangian coordinates makes use of the speed $v$ in relation to the density $\rho$, which is derived from the mean headway spacing $s=1 / \rho$. In Lagrangian coordinates it is possible to explicitly define the number of vehicles per time-space cell. As the KWM is a macroscopic model this can be any number of aggregated vehicles. However a characteristic of the Lagrangian approach also allows one to define a single vehicle per cell.

\section{Advection invariant}

It is well-known that there are differences between drivers and vehicles, which is generally described in the driving behaviour microscopically. Capturing such behaviour macroscopically is less straightforward. The use of Lagrangian coordinates is a game changer in this respect that the numerical scheme follows vehicles rather than time. This allows single vehicles or vehicle groups to be precisely followed in time and allows specific characteristics of these vehicles to propagate with the flow. This propagation of information with a vehicle (group) is described as an advection invariant term. This approach was generalised and explained in traffic flow theory by Lebacque [24] following developments in second order traffic flow theory. The invariant can describe an arbitrary characteristic of traffic flow and describes the drivers specific behaviour in this model and referred to as the vehicle specific invariant, $I$. It is applied in the Lagranian formulation of the KWM with a conservation equation and in the fundamental relation as such:

$$
\begin{aligned}
& \partial_{t} \rho I+\partial_{x}(\rho v I)=0 \\
& v=V(\rho, I)
\end{aligned}
$$

Here the invariant, $I$, is the vehicle specific invariant, a term that denotes a vehicle dependent adjustment factor that directly influences the critical density $\rho$ for each vehicle or group of vehicles depending on the level of discretisation:

$$
\begin{aligned}
& \rho_{\text {crit }}=I \rho_{\text {crit } .0} \\
& \rho_{\max }=I \rho_{\max .0}
\end{aligned}
$$

Where $\rho_{\text {crit }}$ is the critical density and $\rho_{\text {crit. } 0}$ is the deterministic critical density

$\rho_{\max }$ is the jam density and $\rho_{\max .0}$ is the deterministic jam density

The vehicle specific invariant, $I$, is dependent on two parameters that describe the characteristics of specific vehicles in traffic. A more detailed description is not given in 
this contribution. It is however relevant to mention that the value of $I$ will vary around the value 1 with the deviation depending on the parameter settings. Furthermore in this contribution the value of $I$ remains identical for each vehicle (group) and does not change in time of with traffic state. This simplification does not affect the demonstration of the bounded acceleration in the contribution.

\section{Bounded Acceleration}

The concept of bounded acceleration involves a limitation of vehicle capabilities in a model, such that it resembles the capabilities of real traffic flow. The formulation applied here resemble that described in [9] with some adjustments. The KWM conservation equation given in Eq. 5 remains valid, while a limitation is given to the fundamental relation:

$$
\begin{aligned}
& v=\min \left(V(\rho, I), V_{B A}\right) \\
& V_{B A}=v_{t-1}+a_{\max } \Delta t
\end{aligned}
$$

Where $V_{B A}$ is the bounded speed for a vehicle (group) at a certain time and space

$v_{t-1}$ is the speed of the considered vehicle (group) in the previous time step

$a_{\max }$ is the maximum acceleration allowed, while $\Delta t$ indicates the time step

The speed that is then applied to calculate the new location of the vehicle (group) in the numerical scheme in the Lagrangian formulation may therefore be limited when a vehicle (group) has the possibility to accelerate faster than the maximum acceleration rate, $a_{\max }$. This approach therefore does not make changes to the numerical scheme, but rather the input of the vehicles speed into the scheme.

\section{EXPERIMENTAL CASE}

\section{A. Network and model demonstration}

An experimental case is carried out for an 11 kilometre highway corridor with a single bottleneck location. The corridor is modelled in a first order kinematic wave model (KWM) in Lagrangian coordinates with three lanes, with the bottleneck set for a capacity reduction of $20 \%$. The KWM and numerical scheme allow for unconstrained overtaking of vehicles in case of a predecessor catching up with another vehicle group. Traffic flow into the corridor is initially set at an increasing rate from 1000 up to $3000 \mathrm{veh} / \mathrm{hr} / \mathrm{lane}$ and retracts to $2000 \mathrm{veh} / \mathrm{hr} / \mathrm{lane}$ at a set time. The driver specific characteristics of the traffic flow, mean that congestion may occur sooner for a vehicle specific invariant value lower than 1.0 and less readily for values above 1.0. The vehicle specific invariant is set rather liberally such that values between 0.8 1.2 are possible, with one scenario also allowing values between 0.6-1.4. Assignment of invariant values is carried out randomly, using the same random seed for all scenarios. The time step applied in the simulations is 2.0 seconds, while the maximum speed limit is set at $100 \mathrm{kph}$, which meets the CFL condition and eliminates any numerical diffusion issues.
Different scenarios are modelled in which two variables are varied, namely the acceleration bound $\left[\mathrm{m} / \mathrm{s}^{2}\right]$ and the invariant value. The considered scenarios are given in Table 1 .

\begin{tabular}{|c|c|c|}
\hline Scenario number & $\begin{array}{l}\text { Acceleration bound } \\
{\left[\mathrm{m} / \mathrm{s}^{2}\right]}\end{array}$ & Invariant bounds \\
\hline 0 (reference) & $\mathrm{n} / \mathrm{a}$ & $\mathrm{n} / \mathrm{a}(1.0)$ \\
\hline 1 & 2.0 & $\mathrm{n} / \mathrm{a}(1.0)$ \\
\hline 2 & $\mathrm{n} / \mathrm{a}$ & $0.8-1.2$ \\
\hline 3 & 2.0 & $0.8-1.2$ \\
\hline 4 & 2.0 & $0.6-1.4$ \\
\hline 5 & 1.0 & $\mathrm{n} / \mathrm{a}(1.0)$ \\
\hline 6 & 1.0 & $0.8-1.2$ \\
\hline 7 & 0.75 & $0.8-1.2$ \\
\hline 8 & 0.5 & $0.8-1.2$ \\
\hline
\end{tabular}

Table 1 Scenario variable values

The results of the scenarios are given in the form of flowdensity fundamental diagrams, which allow insight into the spread of traffic values. Selected trajectory-space-time plots are given for relevant scenarios and a final comparison of the different levels of bounded acceleration are shown in a cumulative flow diagram.

\section{B. Traffic flow and congestion}

The introduction of vehicle specific invariant values was previously introduced to increase realism in modelling, especially aiding stochastic breakdown. Here bounded acceleration is added to the model. In Figure 1 the density/trajectory plots are given of selected scenarios in which the consequence of various values of the varied variables are shown. The selection is made taking into consideration that the outcome of scenarios 0,1 and 5 were nearly identical, as were scenarios 2,3 and 6 , which were also similar.

The reference scenario 0 shows traffic flow increasing until at a certain point congestion is triggered. Each vehicle group homogenously transverses with the presiding flow and density values without fluctuations in flow. In scenario 2 and 4, the invariant is randomly applied (with an identical random seed for both for comparisons sake). The differences between vehicle groups is obvious and also makes is easy for one to see the trajectories of traffic, especially when a vehicle group reaches a congested road section. More importantly higher bandwidths for the invariant show greater degrees of congestion. This can be seen between scenario 4 , 2 and 0 . In scenario 2 and 8 the same invariant values are applied, however with different bounded acceleration. In scenario 8 an extreme and unrealistic value of $0.5 \mathrm{~m} / \mathrm{s}^{2}$ is applied. This leads to a much denser congestion and longer congestion. However when applying a value of $1.0 \mathrm{~m} / \mathrm{s}^{2}$ to the same case (not shown) little difference was found in the degree of congestion compared to a value of $2.0 \mathrm{~m} / \mathrm{s}^{2}$. The following subsection looks into this in more detail. 


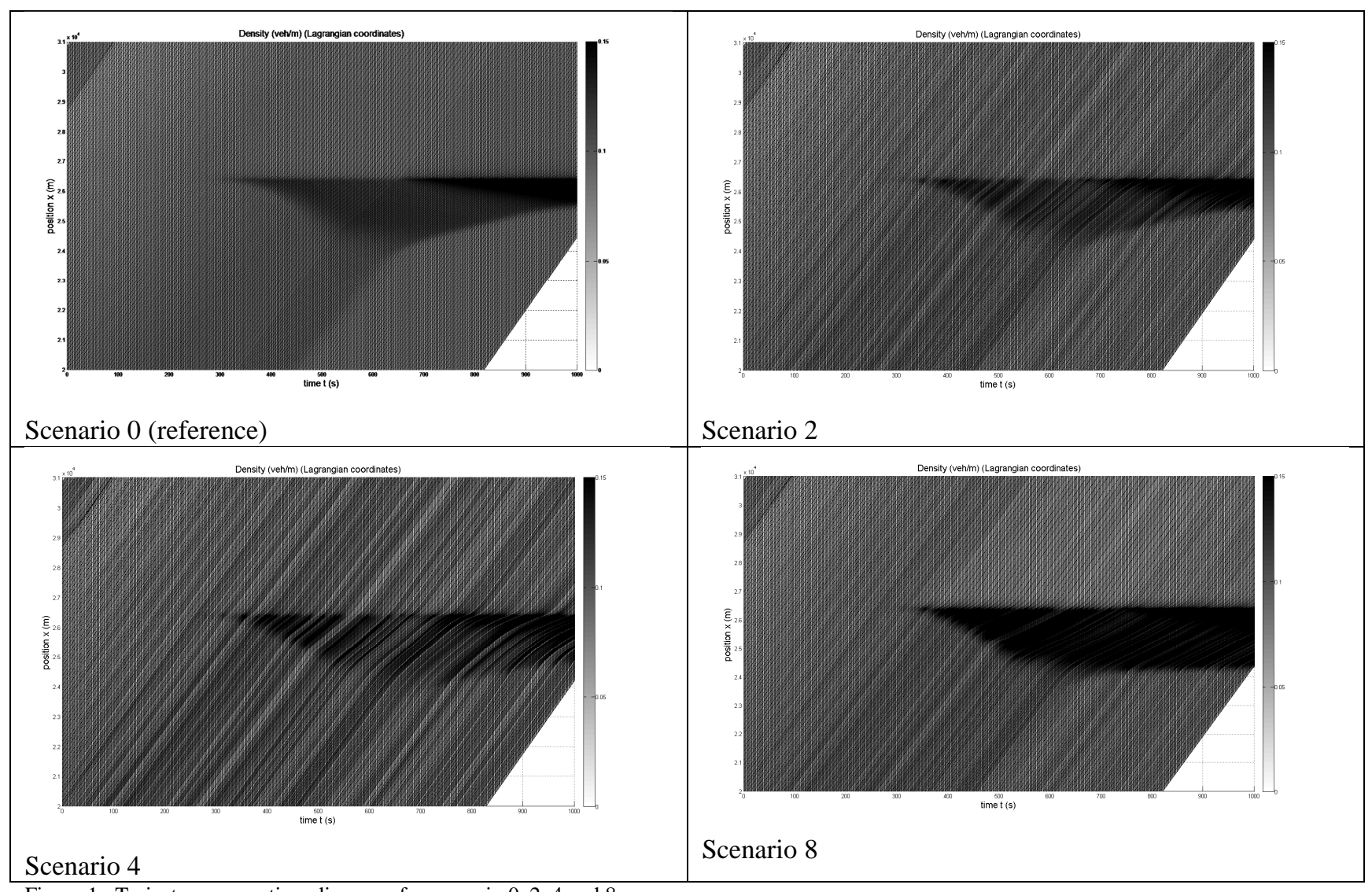

Figure 1 Trajectory-space-time diagrams for scenario $0,2,4$ and 8 .

The fundamental diagrams for each scenario are shown in Figure 3. These are captured at a location directly upstream of the bottleneck. This gives further insight into the spread of traffic flow values and the extent of congestion. A first obvious observation is the spread in points on the congested arm for scenarios with invariant values (scenarios 2,3,4,6,7 and 8). As the invariant is setup to allow 'more aggressive' vehicle groups to drive at a smaller time headway (larger density), a higher flow rate can be achieved for identical speeds, while for 'less aggressive' vehicles the opposite is the case. This can be further noted from the greater spread for scenario 4 with the higher invariant bounds. The resulting fundamental diagrams represent the 'cloud' seen from empirical representations more realistically than the straight lines seen form scenarios 0,1 and 5 without the invariant. The results of the bounded acceleration are further described in the following subsection. Another observation from Figure 3 is the severity of congestion, which is represented by the resulting densities. In the case of a broader invariant value, congestion is found to be slightly more severe, while for very low acceleration bounds an even higher density is found.

\section{A. Bounded acceleration}

Bounded acceleration (BA) is tested in the experimental case in four scenarios $(3,6,7$ and 8$)$ for which the acceleration is bounded at $2.0,1.0,0.75$, and $0.5 \mathrm{~m} / \mathrm{s}^{2}$. A realistic value for road vehicles lies between 1.0 and $2.0 \mathrm{~m} / \mathrm{s}^{2}$, and therefore the values below 1.0 are more demonstrative rather than realistic. From Figures 1 and 3 the effect of BA on the traffic throughput in congestion remained very limited for the two scenarios with 2.0 and 1.0. This is further demonstrated in Figure 2, in which the cumulative number of vehicle groups that pass the bottleneck location are shown in time. A lower number of vehicles indicates that capacity is relatively low and therefore a greater capacity drop is present. Only a very marginal difference is found between an acceleration bound of 2.0 and $1.0 \mathrm{~m} / \mathrm{s}^{2}$. In the hypothetical case that the BA is set to $0.75 \mathrm{~m} / \mathrm{s}^{2}$ and $0.5 \mathrm{~m} / \mathrm{s}^{2}$ an increasingly lower throughput is found in the flow.

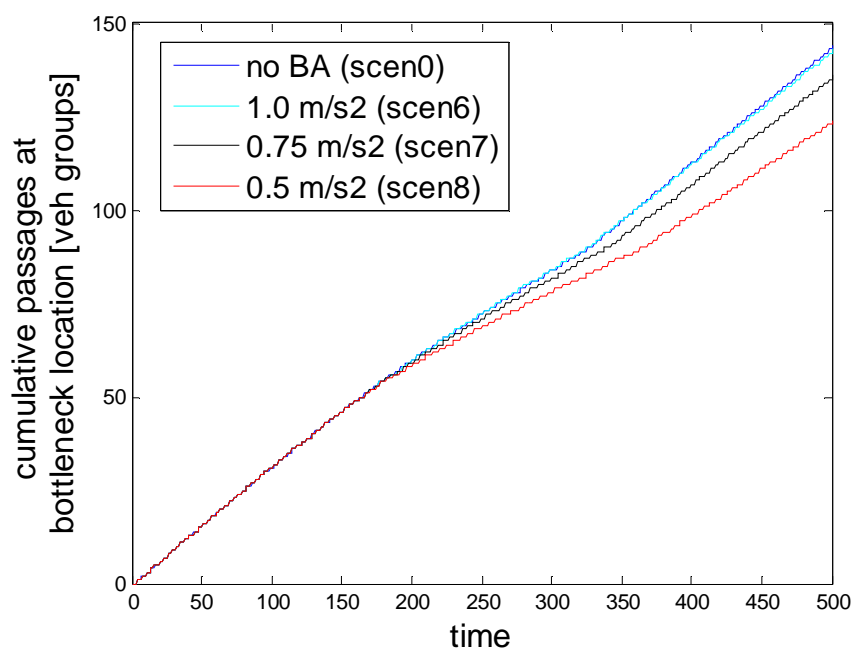

Figure 2 Cumulative throughput at the bottleneck location 


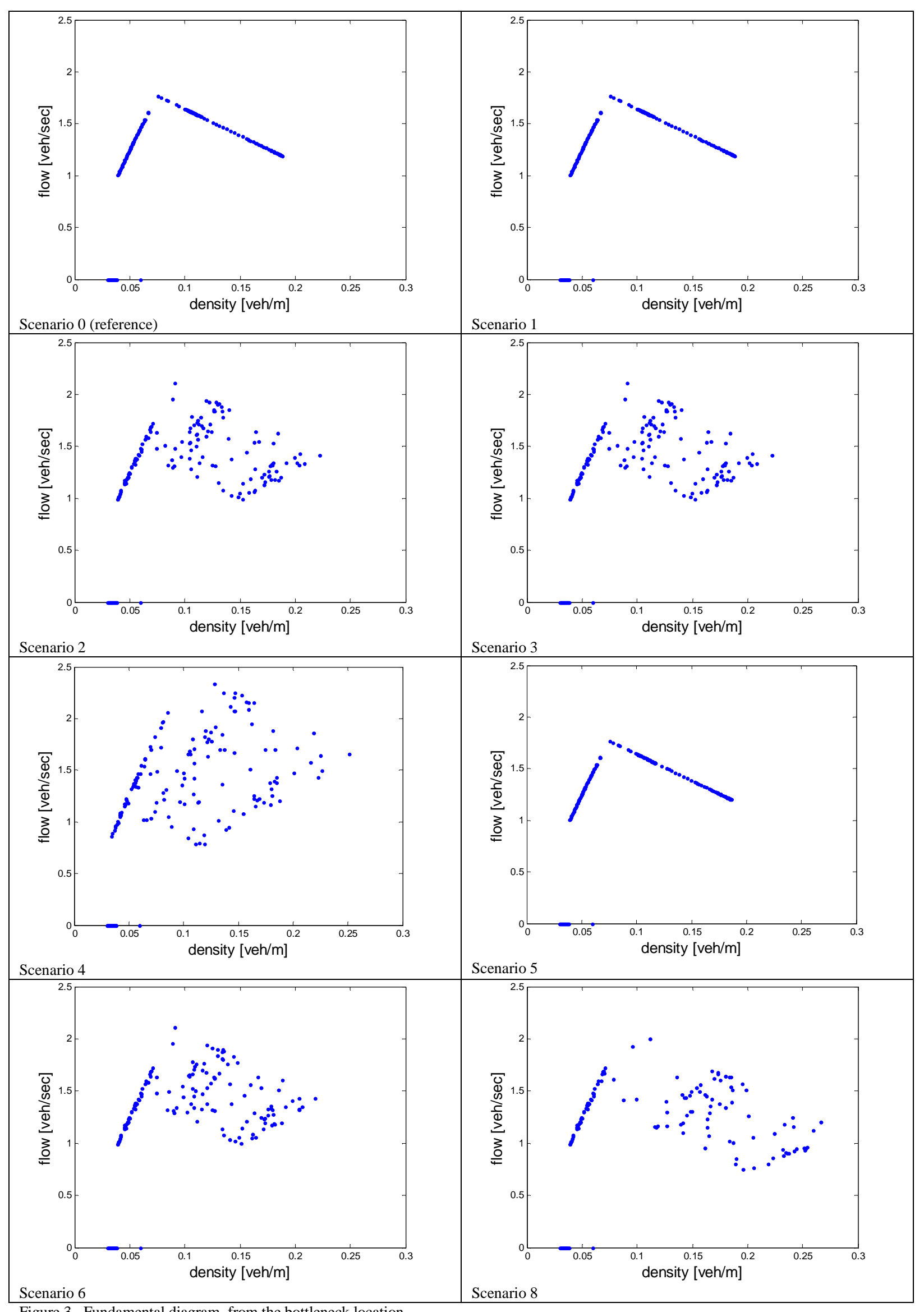

Figure 3 Fundamental diagram, from the bottleneck location 
From these results it can be concluded that the application of bounded acceleration in a driver-specifically modelled flow with unconstrained overtaking does not lead to a substantial capacity drop for realistic values of BA. A drop in throughput is found for more extreme values, which does indicate that BA does directly contribute to some extent, however this is not large enough to be able to contribute the drop to BA of individual vehicle (groups). Therefore a further hypothesis is constructed that the interaction of vehicle characteristics in a constrained manner is more important to reproducing the capacity drop from bounded acceleration. This is therefore also recommended as further research.

\section{CONCLUSION}

This paper gives a demonstration of bounded acceleration in a kinematic wave model formulated in Lagrangian coordinates with driver-specific characteristics and further offers an exploration of the application of bounded acceleration in traffic for the occurrence of the capacity drop where unconstrained overtaking is permitted. The demonstration of the model offers a feasible configuration of bounded acceleration to further improve the realism of the described first order model. The investigation of bounded acceleration found that the application in the model under constrained conditions has a very minor contribution to the capacity drop. Only under extremely low acceleration bounds was there a substantial capacity drop visible. This leads to the conclusion that the capacity drop is not merely a consequence of a restriction in the accelerative ability of vehicles on an individual basis, when vehicles are unconstrained by surrounding vehicles and are easily able to overtake one another. Therefore a further hypothesis is constructed that the interaction of vehicle characteristics in a constrained manner is more important to reproducing the capacity drop from bounded acceleration. It is therefore recommended that further research is carried out for (semi-) constrained multiclass traffic flow in which bounded acceleration is considered. Consideration of dependency between vehicles together with varying acceleration characteristics may lead to the desired capacity drop found on roads in practice.

\section{ACKNOWLEDGEMENTS}

This research is jointly funded by TNO, Netherlands Organisation for Applied Scientific Research, and TrafficQuest, a joint collaboration between TNO, Delft University of Technology, and Rijkswaterstaat, highway agency of the Dutch Ministry of Infrastructure and Environment.

\section{REFERENCES}

M. J. Lighthill and G. B. Whitham, "On Kinematic Waves .2. A Theory of Traffic Flow on Long Crowded Roads," Proceedings of the Royal Society of London Series a-Mathematical and Physical Sciences, vol. 229, pp. 317-345, 1955.

[2] P. I. Richards, "Shock waves on the highway," Operations research, vol. 4, pp. 42-51, 1956.
[3] A. Aw and M. Rascle, "Resurrection of "second order" models of traffic flow," Siam Journal on Applied Mathematics, vol. 60, pp. 916-938, Mar 212000.

[4] C. F. Daganzo, "Requiem for second-order fluid approximations of traffic flow," Transportation Research Part BMethodological, vol. 29, pp. 277-286, Aug 1995.

[5] C. F. Daganzo, "The cell transmission model, part II: network traffic," Transportation Research Part B: Methodological, vol. 29, pp. 79-93, 1995

[6] H. J. Payne, "Models of freeway traffic and control," Mathematical models of public systems, 1971.

[7] H. M. Zhang, "A non-equilibrium traffic model devoid of gaslike behavior," Transportation Research Part B: Methodological, vol. 36, pp. 275-290, 2002.

[8] L. Leclercq, "A new numerical scheme for bounding acceleration in the LWR model," in 4th IMA International Conference on Mathematics in Transport, 2007.

[9] J. Lebacque, "Two-phase bounded-acceleration traffic flow model: analytical solutions and applications," Transportation Research Record: Journal of the Transportation Research Board, vol. 1852, pp. 220-230, 2003.

[10] J. Lebacque, "Intersection modeling, application to macroscopic network traffic flow models and traffic management," in Traffic and Granular Flow'03, ed: Springer, 2005, pp. 261-278.

[11] L. Leclercq, "Bounded acceleration close to fixed and moving bottlenecks," Transportation Research Part B: Methodological, vol. 41, pp. 309-319, 2007.

[12] L. Leclercq, J. A. Laval, and N. Chiabaut, "Capacity drops at merges: An endogenous model," Transportation Research Part B: Methodological, vol. 45, pp. 1302-1313, 2011.

[13] J. W. C. van Lint, S. P. Hoogendoorn, and M. Schreuder, "FASTLANE New Multiclass First-Order Traffic Flow Model," Transportation Research Record, pp. 177-187, 2008.

[14] D. Helbing and M. Treiber, "Numerical simulation of macroscopic traffic equations," Computing in Science \& Engineering, vol. 1, pp. 89-98, 1999.

[15] F. van Wageningen-Kessels, J. Van Lint, S. Hoogendoorn, and C. Vuik, "Implicit time stepping schemes applied to the kinematic wave model in Lagrangian coordinates," in Proceedings of traffic and granular flow, 2009.

[16] L. Leclercq, J. A. Laval, and E. Chevallier, "The Lagrangian coordinates and what it means for first order traffic flow models," in Transportation and Traffic Theory 2007. Papers Selected for Presentation at ISTTT17, 2007.

[17] J. A. Laval, "Hybrid models of traffic flow: impacts of bounded vehicle accelerations," University of California, Berkeley, 2004.

[18] A. Srivastava and N. Geroliminis, "Empirical observations of capacity drop in freeway merges with ramp control and integration in a first-order model," Transportation Research Part C: Emerging Technologies, vol. 30, pp. 161-177, 2013.

[19] J. Banks, "The two-capacity phenomenon: some theoretical issues," Transportation Research Record, 1991.

[20] C. F. Daganzo, M. J. Cassidy, and R. L. Bertini, "Possible explanations of phase transitions in highway traffic," Transportation Research Part A: Policy and Practice, vol. 33, pp. 365-379, 1999.

[21] F. L. Hall and K. Agyemang-Duah, "Freeway capacity drop and the definition of capacity," Transportation Research Record, 1991.

[22] P. S. Farrell, "The hysteresis effect," Human Factors: The Journal of the Human Factors and Ergonomics Society, vol. 41, pp. 226-240, 1999.

[23] F. van Wageningen-Kessels, H. van Lint, S. P. Hoogendoorn, and K. Vuik, "Lagrangian formulation of multiclass kinematic wave model," Transportation Research Record: Journal of the Transportation Research Board, vol. 2188, pp. 29-36, 2010.

J.-P. Lebacque, S. Mammar, and H. H. Salem, "Generic second order traffic flow modelling," in Transportation and Traffic Theory 2007. Papers Selected for Presentation at ISTTT17, 2007. 